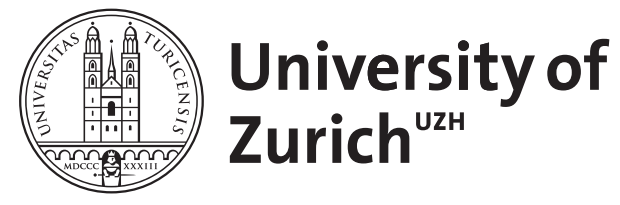

Zurich Open Repository and Archive

University of Zurich

University Library

Strickhofstrasse 39

CH-8057 Zurich

www.zora.uzh.ch

Year: 2012

\title{
Arbeit in Schichtsystemen
}

Gotzmann, M

DOI: https://doi.org/10.1024/1661-8157/a000832

Posted at the Zurich Open Repository and Archive, University of Zurich ZORA URL: https://doi.org/10.5167/uzh-71615

Journal Article

Accepted Version

Originally published at:

Gotzmann, M (2012). Arbeit in Schichtsystemen. Praxis, 101:151-159.

DOI: https://doi.org/10.1024/1661-8157/a000832 


\section{DOI 10.1024/1661-8157/a000832}

((CME PX 3/12))

\section{Arbeit in Schichtsystemen}

Zur Optimierung der Ausnutzung von Produktionsstätten und zur Erweiterung des Dienstleistungssektors arbeiten immer mehr Menschen in Schichtsystemen.

Gemäss Arbeitsgesetz (ArG) der Schweiz liegt Schichtarbeit vor, «wenn zwei oder mehrere Gruppen von Arbeitnehmern und Arbeitnehmerinnen nach einem bestimmten Zeitplan gestaffelt und wechselweise am gleichen Arbeitsplatz zum Einsatz gelangen». Fällt die Arbeit in den Zeitraum von 23 bis 6 Uhr, liegt Nachtarbeit vor [1]. In der Schweiz waren in 2005 bereits $24 \%$ der Arbeitnehmenden in Vollzeit von Nachtarbeit betroffen. Damit gehört die Schweiz in Europa zu den Spitzenreitern [2].

Die möglichen gesundheitlichen Auswirkungen der Schicht- und Nachtarbeit wurden vielfältig untersucht, jedoch sind die Ergebnisse oft unbefriedigend. Sicher ist, dass der Arbeitnehmende in Schichtsystemen unsere besondere Aufmerksamkeit als Patient benötigt, weshalb in den folgenden Kapiteln nachstehenden Fragen bearbeitet werden sollen:

- Was kann die Arbeit im Schichtsystem bewirken und wie können diese Auswirkungen reduziert werden?

- Wie sind die Zusammenhänge von Erkrankungen und der Arbeit im Schichtsystem zu bewerten?

- Ist der Einsatz von Medikamenten hilfreich?

- Gibt es Untersuchungsempfehlungen?

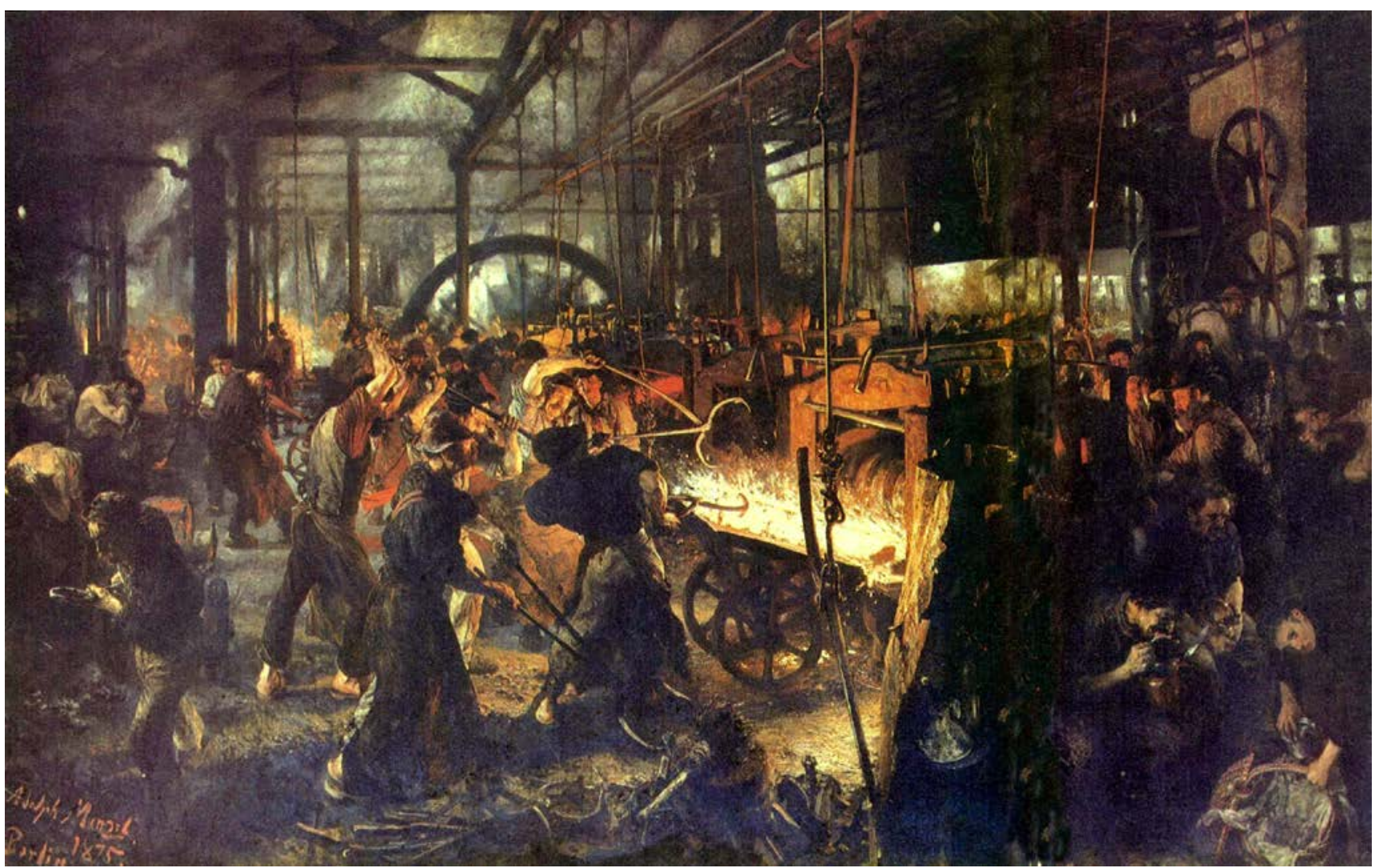

Abb. 1: Eisenwalzwerk von Adolph von Menzel. (Quelle: Wikipedia) 
Ursachen und Auswirkungen von Arbeit in Schichtsystemen

\section{Zirkadianer Rhythmus ((Ü2))}

Der Mensch unterliegt einem zirkadianen Rhythmus der individuell zwischen 22-25 Stunden dauert. Dabei lassen sich chronobiologische Morgen- und Abendtypen unterscheiden. Dieser Rhythmus ist das Ergebnis eines komplexen Wechselspiels von Taktgebern auf molekularer Ebene, den zirkadianen Clock-Genen, einem hierarchischen System mit dem Master - Zeitgeber im Hypothalamus (Nucleus suprachiamaticus), der über endokrine und neuronale Signale einwirkt und äusseren Faktoren, insbesondere dem Tageslicht.

Der Master-Zeitgeber im Nucleus suprachiasmaticus wird mit dem Tageslicht synchronisiert, indem lichtempfindlichen Ganglienzellen der Retina, die das Photopigment Melanopsin enthalten, lichtinduzierte Aktivität auf die Neurone des Master-Zeitgebers vermitteln.

Ein wichtiger Transmitter des zirkadianen Rhythmus ist Melatonin, das in der Epiphyse synthetisiert wird und sehr stark vom Hell-Dunkel-Rhythmus abhängig ist. Die Melatoninsynthese wird bereits ab Beleuchtungsstärken von 30-50 Lux gesenkt und erreicht die maximale Hemmung bei 1000-2000 Lux (Zum Vergleich: Kerze in $1 \mathrm{~m}$ Entfernung $\approx 1$ Lux; Zimmergrundbeleuchtung $\approx 500$ Lux). Dabei ist besonders Licht mit kurzen Wellenlängen (420-480 nm; blaues Licht) wirksam. Allerdings ist das Melatoninsystem eher träge. Bei Jetlag-Untersuchungen konnte eine Verschiebung des individuellen Melatoninspiegelverlaufes pro Tag von maximal 1-1,5 Stunden festgestellt werden [3]. Untersuchungen an Dauernachtarbeitern zeigten nur in <3\% komplette Anpassung und zu $<25 \%$ eine Teilanpassung des Melatoninspiegelverlaufes [4].

\section{Zirkadiane Desynchronisation ((Ü2))}

Eine Abweichung vom eigenen Rhythmus durch äussere Erfordernisse stört dieses komplexe System. Je grösser nun die Diskrepanz zwischen der «inneren Uhr» eines Menschen und den äusseren Taktgebern ist, umso stärker die zirkadiane Desynchronisation. Insgesamt fällt den inneren Zeitgebern eine Phasenverlängerung leichter als eine Phasenverkürzung. Was bedeutet, dass ein chronobiologischer Abendtyp im Spätdienst keine Probleme haben dürfte und einen Nachtdienst über die Verlängerung seines natürlichen Rhythmus besser vertragen wird als beim Morgentyp, der seinen natürlichen Rhythmus stärker verlängern muss und mehr Probleme beim Vorschlafen hat, da dies eine Phasenverkürzung erfordern würde.

$\mathrm{Ob}$ es nun zu einer gut kompensierbaren Irritation der inneren Uhr kommt oder bestehende gesundheitliche Störungen gefördert oder neue hervorgerufen werden können, ist eine Frage des Ausmasses der Phasendiskrepanz und des ununterbrochenen Fortdauerns der Desynchronisation durch die Schichtarbeit.

\section{Mögliche gesundheitliche Auswirkungen ((Ü2))}

Die Ansichten zu Häufigkeiten und Schwere der durch Arbeit in Schichtsystemen induzierten Symptome sind sehr unterschiedlich. Die Gründe liegen in der Schwierigkeit der Expositionserfassung durch unterschiedliche Definitionen und immense Unterschiede in der Gestaltung von Schichtplänen sowie methodischen Problemen. Am Beispiel des Brustkrebs soll dies verdeutlicht werden: Bereits 1987 wurde die Hypothese aufgestellt, dass Melatonin einen Einfluss auf den Brustkrebs hat. Viele Studien folgten mit teilweise divergierenden Ergebnissen. 2007 stufte eine Expertengruppe der International Agency for Research on Cancer (IARC) Schichtarbeit, die den zirkadianen Rhythmus stört, als wahrscheinlich kanzerogen beim Menschen ein. In der Folge wurde Brustkrebs in Dänemark als Berufserkrankung in 38 Fällen anerkannt, bei mehr als 20 Jahren Tätigkeit mit Nachtdienst und Fehlen anderer Risikofaktoren. Andere Studien insbesondere der letzten Zeit relativierten diese Erkenntnisse, so 
dass weitere Untersuchungen erforderlich sind, in denen insbesondere die Exposition der Schichtarbeit, der Chronotypus und die Kofaktoren besser erfasst werden müssen, um zu einer klaren Aussage zu kommen [5].

Durch die zirkadiane Desynchronisation tritt bei Schicht- und Nachtarbeit zunächst das Dilemma einer Leistungsanforderung bei bestehendem Schlafdruck auf, andererseits das Schlaferfordernis zu einer Zeit, in die der Körper auf Aktivität eingestellt ist, so dass der Schlaf nicht das erforderliche Mass abdeckt. Je nach Länge und Ausmass der Desynchronisation können Ein- und/oder Durchschlafstörungen entstehen mit begleitender starker Müdigkeit in den Wachphasen. Bei der Diagnose der Schlafstörung bei Schichtarbeit sind die Zuordnung des chronobiologischen Typus, der Schichtplan und das Schlaftagebuch von entscheidender Bedeutung. Die Kriterien der Diagnose sind im Kasten 1 zusammengefasst.

\section{((Ruedi: Bitte als Kasten gestalten. Merci!))}

Kasten 1: International Classification of Sleep Disorders - Circadian Rhythm Sleep Disorders, Shift Work Type [6].

- Schlaflosigkeit oder exzessive Müdigkeit, die mit Schichtsystemen assoziiert sind, die die übliche Schlafenszeit überlagern,

- Symptome sind mit der Schichtarbeit für mindestens einem Monat verknüpft,

- Für mindestens sieben Tage wird die zirkadiane oder Schlafzeitverschiebung aufgezeichnet (Schlaftagebuch),

- Die Schlafstörungen sind nicht durch andere Beschwerden, Medikamenten - oder Substanzmissbrauch zu begründen.

Neben den Faktoren, die eine zirkadiane Desynchronisation bewirken, kann das Verhalten des Arbeitnehmenden in der Nacht- und Schichtarbeit diese noch verstärken. Dies gilt besonders für die Nahrungsaufnahme, die ein wichtiger dezentraler Zeitgeber ist, der nicht nur vom Zeitpunkt der Aufnahme sondern auch der Zusammensetzung der Nahrung bestimmt wird. Insbesondere bei gastrointestinalen Beschwerden, wie peptischen Ulzera und der Adipositas kann dies von Bedeutung sein, wobei auch Kofaktoren wie Helicobacter pylori, Kaffeegenuss und Rauchen eine Rolle spielen.

\section{Vorerkrankungen und Schichtarbeit ((Ü2))}

In der Praxis stehen wir vor der Frage, ob und welche Schwierigkeiten auftreten können, wenn Patienten mit Vorerkrankungen eine Schichtarbeit aufnehmen wollen oder bei Schichtarbeitenden eine Erkrankung neu auftritt. Dabei sind folgende Aspekte zu betrachten:

- Kann sich eine bestehende Erkrankung oder deren Therapie durch die Schichtarbeit verschlechtern? Hier ist besonders an Erkrankungen zu denken, die vom zirkadianen Rhythmus beeinflusst werden und an Erkrankungen, die eine Medikation mit definiertem Spiegelverlauf erfordern.

- Kann die bestehende Erkrankung ein Sicherheitsrisiko für den Patienten oder den Betrieb darstellen? Die Arbeitsabläufe im Tages- oder Nachtdienst können je nach Arbeitsplatz deutlich unterschiedlich sein. Arbeitet man tagsüber noch im Team, kann der Arbeitnehmer in der Nacht alleine sein. Eine plötzliche gesundheitliche Beeinträchtigung kann nachts deshalb völlig andere Konsequenzen und Risiken zeitigen als im Tagdienst.

Am Beispiel eines Mitarbeitenden mit insulinpflichtigem Diabetes ist dies zu verdeutlichen. Um die Einsatzfähigkeit im Schichtsystem zu beantworten, sind Informationen über die Stabilität der Blutzuckereinstellung, die Fähigkeit der Selbstmessung und Dosisanpassung des Mitarbeitenden, Informationen zum Schichtsystem (siehe unten) und zum Arbeitsplatz (Pausen, Kollegen, Gefährdungen, Aufgaben) 
erforderlich. Wenn Unsicherheiten bestehen, sollte die Möglichkeit eines gut begleitenden Testlaufes überdacht werden, bevor Entscheidungen getroffen werden, die die Anstellung des Arbeitnehmenden ausschliessen.

Grundsätzlich ist die Frage des Einsatzes im Schicht- und Nachtdienst individuell zu stellen und unter Berücksichtigung der Erkrankung, der erforderlichen Therapie und der Situation am Arbeitsplatz zu entscheiden. Bei Unsicherheiten empfiehlt sich die Kontaktaufnahme zu einem Arbeitsmediziner. 


\section{Untersuchung und Beratung von Schichtarbeitenden}

Aufgrund der möglichen Gefährdungen durch Nachtarbeit schreibt der hiesige Gesetzgeber

Angebotsuntersuchungen für Nachtarbeitnehmende vor, die mehr als 25 Nächte im Jahr arbeiten. Für bestimmte Gruppen von Arbeitnehmern kann die medizinische Untersuchung obligatorisch erklärt werden. Den Inhalt und die Durchführung dieser Untersuchungen sind vom Staatssekretariat für Wirtschaft SECO festgelegt. Der Arbeitgeber trägt die Kosten der medizinischen Untersuchung und der Beratung, soweit nicht ein Versicherer des Arbeitnehmers dafür aufkommt. Voraussetzung, Umfang der Untersuchung und Formulare sind auf der Seite der SECO definiert [7].

\section{Analyse des Schichtplanes ((Ü2))}

Um die Belastung des Arbeitnehmenden durch die Arbeit im Schichtsystem einschätzen zu können, sollte dieses zunächst analysiert werden. Schichtpläne werden durch folgende Kennzeichen beschrieben:

- Beginn und Ende der Schichten: Dabei sind die Tageszeiten und eine mögliche Kollision mit der «Inneren Uhr» des Patienten zu beachten.

- Länge der Schichten: Neben der Dauer sind auch die Pausenregelungen und die Möglichkeit eines Nickerchens zu erfassen.

- Nachschicht: Ist sie erforderlich?

- Schichtrotation: Vorwärtsrotation (mit der Uhr) heisst ein Wechsel von Früh-, in den Spät- und in die Nachtschicht. Diese Variante wird empfohlen, da sie im Allgemeinen besser verträglich ist. Bei der Rückwärtsrotation (gegen die Uhr) erfolgen die Wechsel in umgekehrter Reihenfolge; also von der Frühschicht in die Nacht - und dann in die Spätschicht.

- Schnelligkeit der Rotation: Dabei ist die Anzahl der aufeinanderfolgenden gleichen Schichten anzuschauen, insbesondere der Schichten, die Einflüsse auf die «innere Uhr» des Patienten haben und ein akkumuliertes Schlafdefizit hervorrufen können.

Bei der Bewertung kann jedes Kriterium eine Rolle spielen und ist im Zusammenhang mit dem individuellen Rhythmus des Patienten zu sehen.

\section{Analyse weiterer Rahmenbedingungen ((Ü2))}

Auch andere Kriterien am Arbeitsplatz, wie die Gestaltung oder anfallende Überzeiten können für die Verträglichkeit von Schichtarbeit eine Rolle spielen, wie auch Faktoren ausserhalb des Arbeitsplatzes. Eine Übersicht gibt Abbildung 2. 
Einflussfaktoren für die Verträglichkeit der Schicht- und Nachtarbeit

\begin{tabular}{|c|c|c|}
\hline $\begin{array}{l}\text { Lebensbedingungen } \\
\text { Anzahl und Alter der Kinder }\end{array}$ & & $\begin{array}{l}\text { Arbeitsbedingungen } \\
\text { Schichtplan }\end{array}$ \\
\hline $\begin{array}{c}\text { Arbeitszeit des Partners } \\
\text { Wohnsituation } \\
\text { Familiäre Unterstützung } \\
\text { Soziale Unterstützung } \\
\text { Einkommen }\end{array}$ & $\begin{array}{c}\text { Individuum } \\
\text { Alter } \\
\text { Geschlecht } \\
\text { Chronotvo } \\
\text { Gesundheitszustand } \\
\text { Schlafgewohnheiten } \\
\text { Fitness }\end{array}$ & $\begin{array}{l}\text { Kompensationen } \\
\text { Flexible Gestaltung } \\
\text { Arbeitsorganisation } \\
\text { Arbeitsbelastung } \\
\text { Gefährdungen }\end{array}$ \\
\hline
\end{tabular}

Abb. 2: Einflussfaktoren für die Verträglichkeit von Arbeit in Schichtsystemen. 
Empfehlungen für Schichtarbeitnehmende

Empfehlungen für Schichtarbeitnehmende sind individuell zu treffen, da sie den Chronotyp des Arbeitnehmenden, den Gesundheitszustand, den Schichtplan sowie die Arbeitsbedingungen berücksichtigen sollten.

Sollte es erforderlich sein, einen Mitarbeitenden von der Schichtarbeit abzuraten oder gar als nicht geeignet einzustufen, ist das Wechselspiel individueller gesundheitlicher Voraussetzung und der Arbeitsbedingungen entscheidend. Eine Krankheitsdiagnose ist dabei nicht massgeblich, sondern lediglich ein Hinweis auf das Erfordernis einer sorgfältigen Abwägung. Sollten Einschränkungen bezüglich des Einsatzes im Schichtbetrieb vorliegen, empfiehlt es sich, den Empfehlungen der SECO zu folgen und entsprechende Formulare zu nutzen, um die Vorgaben der Schweigepflicht und den gesetzlichen Anforderungen zu genügen. Dies gilt insbesondere, wenn ein Kontakt zum Arbeitgeber hergestellt werden muss.

Da es erfahrungsgemäss einfacher ist, beim Verhalten des Arbeitnehmenden anzusetzen, als Schichtpläne im Betrieb zu verändern, zunächst einige Anmerkungen dazu.

Ziel ist den individuellen Biorhythmus durch die Schichtarbeit so wenig und so kurz wie möglich zu stören. Insbesondere in den hellen Jahreszeiten sollte nach Sonnenaufgang auf dem Nachhauseweg nach dem Nachtdienst eine Brille getragen werden, die das kurzwellige Licht rausfiltert, um die etwaige Melatoninproduktion nicht zu unterdrücken. Bei starkem Schlafdruck in der Nacht wenn möglich helle Lichtquellen nutzen. Ob Koffein zur Verbesserung der psychomentalen Fähigkeiten nützlich sein kann, ist heute noch eine offene Frage [8]. Der Einsatz sollte sich aber auf moderate Mengen beschränken und eventuelle Gesundheitsstörungen berücksichtigen.

Das Essverhalten sollte auch bei Schichtwechseln möglichst beibehalten werden. Das bedeutet idealerweise morgens ein leichtes Frühstück, mittags eine kleine Mahlzeit und abends ein leichtes Essen einzunehmen, das insbesondere im Nachtdienst wenig Kohlenhydrate und Fett enthalten sollte. Wenn erforderlich, kann noch ein Spätsnack eingenommen werden, wobei nach Möglichkeit zwischen Mitternacht und 6h morgens nicht gegessen werden sollte.

Eine medikamentöse Beeinflussung des Schlaf-Wachrhythmus des Schichtarbeitenden ist schwierig. Melatonin (Circadin $\AA$ ) ist in der Schweiz nur für die Behandlung der Insomnie bei Patienten ab dem 55. Lebensjahr zugelassen. Auch Modafinil zur Vigilanzsteigerung ist nur für exzessive Schläfrigkeit in Zusammenhang mit Narkolepsie zugelassen und nicht für Schlafstörungen durch Schichtarbeit. Vom Einsatz von Hypnotika ist abzuraten, da sie die Amplitude des endogenen Rhythmus abschwächen und somit einen nachteiligen Effekt haben können. Somit stehen die Verhaltensmassnahmen wie gezielter Umgang mit Licht, Einhalten fester Rhythmen und Schlafhygiene weiterhin im Vordergrund.

Dem Arbeitgeber ist zu empfehlen, die Schichtwechsel vorwärtsrotierend zu gestalten und die Anzahl der kontinuierlichen Schichten, die mit dem individuellen Chronotyp und den Bedürfnissen kollidieren, klein zu halten. Die höchste Verträglichkeit ist durch hohe Flexibilität und Mitgestaltung der Schichtpläne durch die Arbeitnehmenden zu erreichen. 


\section{Fallbericht zur Arbeit in Schichtsystemen}

Y

Anamnese der Patientin

In der arbeitsmedizinischen Sprechstunde stellt sich im Rahmen der betrieblichen Wiedereingliederung eine 34 jährige Pflegefachfrau vor. Vor sechs Monaten wurde sie brusterhaltend operiert, nachdem die Diagnose Brustkrebs gestellt worden war. Eine anschliessende Bestrahlung für fünf Wochen wurde von erheblichen Beschwerden begleitet, von denen sie sich aber langsam erholt hat. Weitere Therapien sind zurzeit nicht notwendig. Die Nachkontrollen finden regelmässig beim behandelnden Frauenarzt statt. Die Patientin hat vor der Erkrankung als Pflegefachfrau im Spital gearbeitet. Da sie alleinerziehende Mutter ist, arbeitete sie bisher als Dauernachtwache mit ca. 50\% Pensum, um die Betreuung ihrer 8-jährigen Tochter sicher zu stellen. In der Regel arbeitet sie zwei bis drei Nächte / Woche von Freitag- bis Sonntagnacht, da ihre Tochter am Wochenende bei ihrem Vater ist.

Aktuell ist sie zu 100\% arbeitsunfähig geschrieben.

\section{Kommentar}

Fragestellung: Rückkehr an den alten Arbeitsplatz möglich?

Zurzeit kurativ behandelter Brustkrebs; Therapie abgeschlossen.

Erhebliche soziale Vorteile durch die bisherige Arbeitsplatzkonstellation.

\section{Weg zur Diagnose/Kommentar}

\section{Status((Ü2))}

Die Patientin arbeitet gerne nachts, da sie ein

«Nachtmensch» ist. Da die Tochter meistens am

Wochenende nicht da ist, kann sie danach auch gut ausschlafen, da ihre Wohnung ruhig liegt.

Die Schichtdauer beträgt 8h 24min. Eine Schichtrotation besteht nicht. Ohne Unterbruch werden zwei bis drei Nachtschichten gearbeitet.

\section{Vermutlicher Chronotyp: Abendtyp}

Ungestörter Tagesschlaf möglich

\section{Empfehlungen}

Wiedereinsatz als Dauernachtwache mit 50\% Pensum.

Eine Wiedervorstellung in sechs Monaten oder bei Auftreten von Beschwerden sollte vereinbart werden.

Empfehlungen zur Ernährung bei Schichtarbeit und zum

Tragen einer Brille mit Blaulichtfilter nach dem Nachtdienst.
Die Patientin ist vom Chronotyp eher für den Nachtdienst geeignet und hat inn auch bisher gut vertragen. Eine Frequenz von zwei bis drei Nächten stellt zwar eine Irritation des Biorhythmus dar, der sich aber in den folgenden Tagen wieder korrigieren sollte. 

und fühlt sich entlastet.

Ganzheitlich gesehen, profitiert die Patienten von der Wiederaufnahme der Tätigkeit.

Sollten auf Grund des veränderten

Gesundheitszustandes der Patienten

Beschwerden auftreten, muss die Situation neu

überdacht werden. 


\section{Fragen zur Arbeit in Schichtsystemen}

Frage 1

Welche Aussage zur Nachtarbeit in der Schweiz trifft zu? (Einfachauswahl, 1 richtige Antwort)

a) Von Nachtarbeit sind nur wenige Arbeitnehmende betroffen, weshalb sie keine praktische Relevanz hat.

b) Die Kriterien der Nachtarbeit sind im Arbeitsgesetz definiert.

c) Nachtarbeit liegt vor, wenn das Tageslicht zur Ausübung der Tätigkeit nicht ausreicht und künstliches Licht ergänzt werden muss.

d) Im Vergleich zu den Nachbarländern kommt Nachtarbeit in der Schweiz deutlich weniger vor.

e) Jeder Mitarbeitende der mindestens $1 \mathrm{x} /$ Monat in der Nacht arbeitet, sollte medizinisch betreut werden.

\section{Frage 2}

Melatonin ist ein wichtiger Transmitter des zirkadianen Rhythmus. Welche Angaben sind richtig? (Mehrfachauswahl, 3 richtige Antworten)

a) Melatonin wird in den lichtempfindlichen Ganglienzellen der Retina gebildet.

b) Die Melatoninsynthese wird bereits ab Beleuchtungsstärken von 30-50 Lux gesenkt und erreicht die maximale Hemmung bei 1000-2000 Lux.

c) Besonders langwelliges Licht ist in der Blockierung des Melatonins effektiv.

d) Beim Jetlag ist eine Verschiebung des individuellen Melatoninspiegelverlaufes pro Tag von maximal 1-1,5 Stunden festgestellt worden.

e) Dauernachtarbeiter zeigen nur in geringem Mass eine komplette Anpassung des Melatoninspiegelverlaufes.

Frage 3

Bei welcher Kombination von biologischem Chronotyp und Schichtanforderung erwarten Sie die grössten Probleme? (Alle weiteren Faktoren der Verträglichkeit werden nicht berücksichtigt) (Einfachauswahl, 1 richtige Antwort)

a) Eine Morgentyp wird im Frühdienst (Beginn 6.15h) eingesetzt.

b) Eine Morgentyp wird im Spätdienst (Ende 22.30h) eingesetzt.

c) Eine Morgentyp wird im Nachtdienst (Beginn 22.10h, Ende 6.34h) eingesetzt.

d) Eine Abendtyp wird im Spätdienst (Ende 22.30h) eingesetzt.

e) Eine Abendtyp wird im Nachtdienst (Beginn 22.10h, Ende 6.34h) eingesetzt.

Frage 4

Welche Auswirkungen von Nacht- und Schichtarbeit auf die Gesundheit sind richtig? (Mehrfachauswahl, 2 richtige Antworten)

a) Schlafstörungen vom Schichtarbeitstyp sind gekennzeichnet durch Schlaflosigkeit oder exzessive Müdigkeit, die mit Schichtsystemen assoziiert sind, die die übliche Schlafenszeit überlagern.

b) Der ursächliche Zusammenhang von Brustkrebs und Arbeit in Schichtsystemen ist gesichert.

c) Bei der Entwicklung der Adipositas von Schichtarbeitenden spielt die Nahrungsaufnahme nur eine untergeordnete Rolle, da der Stoffwechsel überwiegend von zentralen Zeitgebern gesteuert wird. 
d) Der Einsatz von Arbeitnehmenden mit Vorerkrankungen im Schicht- und Nachtdienst ist individuell zu stellen und unter Berücksichtigung der Erkrankung, der erforderlichen Therapie und der Situation am Arbeitsplatz zu entscheiden.

e) Treten gesundheitliche Störungen bei der Arbeit in Schichtsystemen auf, sollte der Arbeitnehmende möglichst lange in der Schicht eingesetzt werden, in der er Probleme hat, damit sich die «innere Uhr» umstellen kann.

\section{Frage 5}

Bei der Beratung und Untersuchung von Schichtarbeitnehmenden ist Folgendes zu beachten:

a) Eine rückwärtsrotierende Schichtplanung ist einer vorwärtsrotierenden vorzuziehen.

b) Ab dem 40. Lebensjahr kann die angebotene medizinische Betreuung von Schichtarbeitenden jährlich wahrgenommen werden.

c) Der Arbeitgeber trägt die Kosten der medizinischen Untersuchung und der Beratung, soweit nicht ein Versicherer des Arbeitnehmers dafür aufkommt.

d) Faktoren, die ausserhalb des Arbeitsplatzes liegen, sind bei der Bewertung der Schichtverträglichkeit unerheblich.

e) Mitarbeitende, die morgens müde zur Frühschicht kommen, sollten eine Brille tragen, die das kurzwellige Licht rausfiltert um die Melatoninproduktion zu unterdrücken.

\section{Korrespondenzadresse}

Dr. med. Margrit Gotzmann

Abteilung für Arbeits- und Umweltmedizin

Universitätsspital Zürich

Rämistrasse 100

8091 Zürich

margrit.gotzmann@usz.ch

\section{Bibliographie}

1. Arbeitsgesetz (ArG) SR 822.11 Art. 17c; Verordnung 1 zum ArG (ArGV1) SR 822.111 Art. 43-45. http://www.admin.ch/ch/d/sr/sr.html2. 4. Europäische Erhebung über die Arbeitsbedingungen 2005 Ausgewählte Ergebnisse aus Schweizer Perspektive. Graf M et al. Staatssekretariat für Wirtschaft SECO 2007.

3. Stuck BA, Maurer JT, Schredl M, Weess HG. Praxis der Schlafmedizin. Springer Heidelberg, 2009.

4. Folkhard S. Do permanent night workers show circadian adjustment? A review based on the endogenous Melatonin rhythm. Chronobiology international 2008; 25: 215-224.

5. Stevens et al. Considerations of circadian impact for defining 'shift work' in cancer studies: IARC Working group report. Occup Environ Med 2011; 68: 154-162.

6. American Academy of sleep medicine. International classification of sleep disorders (2nd ed.): Diagnostic and coding manual. American Academy of Sleep Medicine, Westchester, 2005.

7. Medizinische Untersuchung und Beratung bei Nachtarbeit - Staatssekretariat für Wirtschaft (SECO). http://www.seco.admin.ch/themen/00385/01901/01907/index.html?lang=de 
8. Ker $\mathrm{K}$ et al. Caffeine for the prevention of injuries and errors in shift workers. Cochrane Database of Systematic Reviews 2010, Issue 5. Art.No.: CD008508. 


\title{
Antworten zu den Fragen zur Arbeit in Schichtsystemen aus PRAXIS Nr. 3
}

\author{
Frage 1
}

\section{Antwort b) ist richtig.}

Fällt die Arbeit in den Zeitraum von 23 bis 6 Uhr, liegt Nachtarbeit vor.

ad a) 2005 waren 24\% der Arbeitnehmenden in der Schweiz von Nachtarbeit betroffen.

ad c) siehe b)

ad d) Im Vergleich zu den Nachbarländern ist die Schweiz bei der Häufigkeit der Nachtarbeit Spitzenreiter. ad e) Jeder Mitarbeitende der > 25 Nächte/Jahr arbeitet sollte medizinisch betreut werden.

\section{Frage 2}

Die Antworten b), d) und e) sind richtig.

Die Melatoninsynthese wird bereits ab Beleuchtungsstärken von 30-50 Lux gesenkt und erreicht die maximale Hemmung bei 1000-2000 Lux.

Beim Jetlag ist eine Verschiebung des individuellen Melatoninspiegelverlaufes pro Tag von maximal 1-1,5 Stunden festgestellt worden.

Dauernachtarbeiter zeigen nur in $<3 \%$ eine komplette Anpassung des Melatoninspiegelverlaufes. ad a) Melatonin wird in den Pinealozyten der Epiphyse synthetisiert. In den lichtempfindlichen Ganglienzellen der Retina wird das Melanopsin gebildet.

ad c) Besonders kurzwelliges Licht (420-480 nm; blaues Licht) ist in der Blockierung des Melatonins effektiv.

Frage 3

Antwort c) ist richtig.

Wird der Morgentyp in der Nachtschicht eingesetzt fällt die erforderliche Aktivitätsphase völlig in die biologische Ruhephase. Es besteht maximale zirkadiane Desynchronisation.

ad a) Im Frühdienst wird der Morgentyp eher gut einzusetzen sein, da die biologische Aktivitätsphase früh am Tag beginnt.

ad b) Beim Spätdienst überlagert sich das Ende beim Morgentyp vermutlich bereits mit der biologischen Ruhephase.

ad d) Beim Abendtyp fällt der Spätdienst vermutlich noch in die biologische Aktivitätsphase.

ad e) Beim Abendtyp fällt die Nachtarbeit teilweise noch in die biologische Aktivitätsphase. Nach dem Dienst befindet sich der Abendtyp möglicherweise noch in der biologischen Ruhephase, was den Schlaf erleichtern würde.

Frage 4

Die Antworten a) und d) sind richtig.

Schlafstörungen vom Schichtarbeitstyp sind gekennzeichnet durch Schlaflosigkeit oder exzessive Müdigkeit die mit Schichtsystemen assoziiert sind, die die übliche Schlafenszeit überlagern.

Der Einsatz von Arbeitnehmenden mit Vorerkrankungen im Schicht- und Nachtdienst ist individuell zu stellen und unter Berücksichtigung der Erkrankung, der erforderlichen Therapie und der Situation am Arbeitsplatz zu entscheiden. 
ad b) Der ursächliche Zusammenhang von Brustkrebs und Arbeit in Schichtsystemen ist nicht gesichert.

ad c) Bei der Entwicklung der Adipositas von Schichtarbeitenden spielt die Nahrungsaufnahme nur eine wichtige Rolle, da der Stoffwechsel überwiegend von dezentralen Zeitgebern gesteuert wird.

ad e) Treten gesundheitliche Störungen bei der Arbeit in Schichtsystemen auf, sollte der Arbeitnehmende möglichst wenig in dieser Schicht eingesetzt werden, da selbst bei Dauernachtarbeitenden nur zu einem geringen Prozentsatz eine Anpassung der «innere Uhr» stattfindet.

\section{Frage 5}

Antwort c) ist richtig.

Der Arbeitgeber trägt die Kosten der medizinischen Untersuchung und der Beratung, soweit nicht ein Versicherer des Arbeitnehmers dafür aufkommt.

ad a) Eine vorwärtsrotierende Schichtplanung ist in der Regel verträglicher.

ad b) Ab dem 45. Lebensjahr kann die angebotene medizinische Betreuung von Schichtarbeitenden jährlich wahrgenommen werden.

ad d) Auch Faktoren, die ausserhalb des Arbeitsplatzes liegen, wie familiäre Situation und Lebensbedingungen sind bei der Verträglichkeit von Schichtsystemen zu berücksichtigen.

ad e) Mitarbeitende, die morgens aus der Nachtschicht kommen, sollten eine Brille tragen, die das kurzwellige Licht rausfiltert um die Melatoninproduktion nicht zu unterdrücken. 